\title{
Heart Disease in Adults With Down Syndrome Between 1996 and 2016
}

\author{
Veronica Fitzpatrick, DrPH, MPH, Anne Rivelli, MA, MPH, Kelsey Bria, BS, and \\ Brian Chicoine, MD
}

Purpose: To describe demographic factors and calculate prevalence of heart disease-related conditions among the adult Down syndrome (DS) sample population and to compare demographic and heart disease-related conditions between the DS sample population $(n=2342)$ and the general population.

Methods: Using a retrospective, descriptive cohort study design, analyses were based on 20 years of data collected on the Adult Down Syndrome Center patient population. Prevalence of heart disease, stroke, and associated risk conditions are reported as counts (\%) with corresponding odds ratio (OR) indicating odds of diagnosis among the DS sample compared with the general population. Corresponding Pearson $c^{2} P$-values were calculated to represent statistically significant differences between prevalence of diagnoses in the DS sample compared with the general population. In cases where prevalence was low, Fisher's Exact Test $P$-value were calculated.

Results: Adults with DS had lower odds of diagnosis of heart disease and most associated risk conditions, specifically coronary heart disease $(\mathrm{OR}=0.0537, P<.0001)$, heart failure $(\mathrm{OR}=\mathbf{0 . 6 3 5 3}$, $P=.0091)$, hypertension $(\mathrm{OR}=0.0325, P<.0001)$, diabetes $(\mathrm{OR}=0.4840, P<.0001)$, and high total cholesterol $(\mathrm{OR}=\mathbf{0 . 2 0 8 6}, P<.0001)$, while experiencing higher odds of overweight status $(0 R=1.2185, P=.0002)$ and obese status $(0 R=1.3238, P<.0001)$.

Conclusion: Adults with DS generally experience less heart disease and associated risk conditions commonly seen in the general population. Prevention and treatment guidelines for heart disease for the DS population should be adjusted after more research is conducted. ( $\mathrm{J}$ Am Board Fam Med 2020;33:923-931.)

Keywords: Cardiovascular Diseases, Chronic Disease, Cohort Studies, Down Syndrome, Heart Diseases, Prevalence, Primary Health Care, Preventive Health Services, Retrospective Studies

\section{Introduction}

Down syndrome (DS) is characterized by intellectual disabilities, dysmorphic features, and comorbid conditions that result from increased genetic material of the 21 st chromosome. ${ }^{1-3}$ DS is the most

This article was externally peer reviewed.

Submitted 18 November 2019; revised 21 May 2020; accepted 21 May 2020.

From the Advocate Aurora Health, Downers Grove, IL (VF, AR, BC); James R. and Helen H. Russell Center for Research \& Innovation, Park Ridge, IL (VF, AR); Rosalind Franklin Medical University/Chicago Medical School, North Chicago, IL (KB); Advocate Medical Group Adult Down Syndrome Center, Park Ridge, IL (BC).

Funding: None.

Conflict of interest: None.

Corresponding author: Veronica Fitzpatrick, DrPH, MPH, Russell Center for Research \& Innovation, Advocate Lutheran General Hospital, 1775 Dempster Street, Suite W-939, Park Ridge, IL 60068 (E-mail: Veronica.fitzpatrick@advocate health.com). frequent chromosomal abnormality among liveborn infants and the most common genetic cause of intellectual disability in the world. ${ }^{3-5}$ As recently as 1983, an adult with DS was only expected to live until age 25 years, but with improved screening and advances in medical technology, many individuals with DS are currently living until 60 years. ${ }^{2,6}$ While individuals with DS are living longer, DS now poses new challenges for the individual, families, and clinical care teams, particularly the prevention and treatment of aging-related conditions. Due to limited information on adults with DS and the increasing lifespan of individuals with DS, research into what the clinical trajectory of adults with DS looks like is warranted to improve the health of adults with DS and update current health guidelines. ${ }^{1,2,4,7,8}$

Recent research suggests that heart disease prevalence in individuals with DS is different from the 
general population or than individuals without DS. ${ }^{9}$ It is well known that congenital heart defects and related complications are more common among ndividuals with DS, ${ }^{5}$ but little is known about acquired coronary heart disease (CHD), more broadly, and cardiovascular (CVD) risk factors. ${ }^{10}$ A systematic review of literature related to adults with DS between 1993 and 2008 noted ischemic heart disease as a minor cause of death among older adults, ${ }^{9}$ despite high rates of obesity and sedentary lifestyles in adults with DS ${ }^{11-15}$ and also despite ischemic heart disease being a major cause of death among the general population. ${ }^{9}$ Current evidence from the general population would suggest that those individuals with DS who experienced obesity persisting into adulthood would be at a greater risk of developing $\mathrm{CHD}$, diabetes and the various other diseases for which obesity is a risk factor. ${ }^{14}$

Current research around cardiac disease and DS is rarely comprehensive. Among adults with DS, separate prior literature has identified higher rates of CVD risk factors, such as hypertension, ${ }^{16}$ obesity, ${ }^{11-14}$ and diabetes, ${ }^{11,16}$ but lower rates of atherosclerotic disease ${ }^{16-19}$ and ischemic heart disease, ${ }^{20}$ furthering the hypothesis that individuals with DS present differently in a health care setting compared with individuals without DS or individuals in the general population. Considering the limited research highlighting inherent clinical differences in individuals with DS, further inquiry into the trajectory of heart disease in individuals with DS is critical for health care, especially given the increased life expectancy over the past few decades.

This study explored prevalence of heart disease, stroke, and associated cardiovascular risk factors in a subpopulation of adults with DS from medical records abstracted from a large Illinois-based health care system. Using the disease and risk condition framework presented in the annual American Heart Association (AHA) Report on Heart Disease and Stroke Statistics ("AHA Report"), this study provides prevalence data on heart disease, stroke, and associated risk conditions among a large sample of adults with DS as well as corresponding prevalence data of the US population provided in the 2019 AHA Report. ${ }^{21}$ These US population prevalence statistics represent the most comprehensive, up-to-date national data on heart disease, stroke, and associated risk conditions and the best context currently available in which to understand these conditions among this sample of adults with DS. ${ }^{21}$ Specifically, this study had 2 objectives:
1. Describe demographics and prevalence of heart disease, stroke, and associated risk conditions among a sample of adults with DS using ICD-9/10 codes from outpatient EMRs of a primary care setting.

2. Compare demographics and prevalence of heart disease, stroke, and associated risk conditions between adults with DS and the general population.

\section{Methods}

This study utilized data abstracted from Advocate Lutheran General Hospital (ALGH), which includes the Advocate Medical Group Adult Down Syndrome Center (ADSC), outpatient EMR system, to meet the study objectives.

\section{Advocate Medical Group ADSC at ALGH}

The ADSC at ALGH is the largest DS clinic in the United States. Started in 1992 through a collaboration between the National Association for Down Syndrome and ALGH, ADSC has grown from seeing patients 2 days per month in shared clinic space to seeing patients 5 days per week in a 6500 -square foot facility designed specifically to accommodate individuals with DS. Since 1992, ADSC has seen over 6000 unique patients with DS. Over the past 10 years, ADSC has served approximately 4500 individuals with DS annually, with about 7500 unique patient visits each year.

Geographically, individuals receiving care at ADSC spans 26 total US states, representing 514 total US cities. At ADSC, sex is evenly distributed (52.5\% male, $47.5 \%$ female) with an overall mean age of 32.68 years and a range of 0 - to 80 -year-olds receiving care. Almost all patients at ADSC have insurance, as many people with DS quality for public aid and may also have private insurance. Medically, individuals with DS receiving care at ADSC experience a variety of diagnosed health issues that are commonly seen in the adult DS population.

\section{Participants}

This retrospective, descriptive cohort study utilized 20 years of data collected at a Chicagoland suburban hospital site that is part of a 22-hospital midwestern nonprofit health care organization. This study obtained approval by Advocate Institutional Review Board (\#6190).

For study inclusion, adults ages $\geq 18$ years who resided in the United States and had a primary 
diagnosis of DS diagnosed in an outpatient setting at ALGH/ADSC between January 1996 and June 2016 were identified. DS diagnosis was identified by the International Classification of Diseases ninth revision (ICD-9) diagnosis code of 758.0 or the equivalent 10th revision (ICD-10) diagnosis code of Q90.9 in the Advocate Health Care electronic medical record (EMR) systems. Data analysts identified eligible cases based on inclusion criteria, excluding cases where the individual was younger than age 18 years or did not have a primary encounter diagnosis of DS.

\section{Procedures}

To address the first objective, demographic factors were aggregated, and prevalence of heart disease, stroke, and associated risk conditions were calculated among the DS study sample. Prevalence among the DS study sample was assessed by totaling cases with at least 1 ICD-9 or ICD-10 code in each condition category, with corresponding codes for each condition category listed in the AHA Report. Demographic factors included: age, sex, race, and ethnicity. Per the AHA Report, this study reports on 3 categories of conditions. Heart disease conditions included: CHD, myocardial infarction (MI), angina, and heart failure (HF). While stroke (cerebrovascular disease) is generally classified as a heart disease condition, stroke was categorized separately in this study in line with the AHA Report. Finally, associated risk conditions included: hypertension, diabetes mellitus (DM), weight status, and total cholesterol (TC). Data analysts were provided ICD-9 diagnosis codes for the disease and risk conditions of interest but, in most cases, clinical data were extracted from the ALGH EMR using ICD-10 code equivalents. See Appendix 1 for all diagnosis variables described in this study, including ICD-9 and ICD-10 codes representing the diagnoses if applicable.

To address the second objective, corresponding prevalence representing the general population were pulled from the AHA Report to best compare differences. These heart disease, stroke, and associated risk condition prevalence were gathered using the National Health and Nutrition Examination Survey. For the purposes of this study, general population prevalence data used in this study represents adults ages $\geq 20$ years between 2013 and 2016, as this time span is the most recent summary report with statistics available for the conditions of interest in this study. For overweight status, data represents adults ages $\geq 20$ years between 2011 and 2014 instead of between 2013 and 2016, as these were the most recent that were available. ${ }^{21}$

After general population prevalence numbers were abstracted, odds, odds ratios (OR), and corresponding $P$-values were calculated to compare the DS study sample prevalence data with the general population data. For the purposes of this study, OR represents the likelihood of the condition occurring among the DS sample relative to the general population. Demographic factors representing the general population were 2016 estimates, generated from 2012 to 2016 data from the US Census Bureau's 2016 American Community Survey 5-Year Population Estimates Program statistics on demographic and housing estimates. ${ }^{22}$

\section{Statistical Methods}

Data management and analysis of the DS sample were conducted with SAS statistical software (Version 9.4; SAS Institute, Cary, NC). All analysis of the DS sample data were performed by research personnel at ALGH.

Demographic, heart disease, stroke, and associated risk conditions are reported as counts (\%), with corresponding OR representing the ratio of odds of conditions among the DS sample relative to the general population. Corresponding Pearson $c^{2} P$-values represent statistically significant differences between prevalence of diagnoses in the DS sample relative to the general population. Pearson $c^{2} P$-values were interpreted, except when cell sizes were less than 10 , in which case Fisher's Exact test $P$-values were interpreted. Finally, odds were calculated and presented to provide an understanding of a condition having occurred in the general population relative to the sample with DS.

We calculated sample size to detect a difference between 2 proportions, using CHD as the primary outcome of interest. Per the AHA Report, the most up-to-date prevalence describing CHD in the general population was $6.3 \%$. Assuming a $2 \%$ lower prevalence in the DS sample (4.3\%), 2301 cases were required in the DS Sample to detect a difference in proportions with $99 \%$ power at a 2 -sided $P$-value of .05 . We preserved all eligible cases $(\mathrm{n}=2342)$.

\section{Results}

During the study period (January 1996 to June 2016), a total of 2342 adult patients ages $\geq 18$ years with a primary diagnosis of DS were identified 
using the ICD-10 diagnosis code Q90.9. The DS sample of adults are primarily female $(53.67 \%)$ and white $(80.03 \%)$ of Non-Hispanic or Latino origin (89.45\%). Table 1 displays the demographics of the DS sample and corresponding data representing the general population. General population estimates represent demographic data available in 2016 describing adults ages $\geq 18$ years between 2012 through 2016. General population adults are primarily female $(50.80 \%)$ and white $(73.30 \%)$ of Non-Hispanic or Latino origin (82.70\%).

\section{CHD: Coronary Heart Disease, Acute Coronary Syndrome, and Angina Pectoris (ICD-9 410 to 414, ICD-10 I20-I25, Includes MI ICD-10 I21-I22)}

In accordance with the AHA Report, prevalence of CHD among the DS sample includes Angina and
MI, but prevalence of Angina and MI were also calculated and reported separately.

Adults in the DS sample have 0.9463 lesser odds of experiencing CHD compared with the general population $(P<.0001)$, highlighting 18.62 :1 odds of experiencing CHD among adults in general population compared with adults with DS. Adults in the DS sample have 0.9771 lesser odds of experiencing angina compared with the general population $(P<.0001)$, highlighting 43.69:1 odds of experiencing angina among adults in general population compared with adults with DS. Adults in the DS sample have 0.9862 lesser odds of experiencing MI compared with the general population $(P<.0001)$, highlighting 72.40:1 odds of experiencing $\mathrm{MI}$ among adults in the general population compared with adults with DS.

Table 1. Demographics among Down syndrome (DS) Sample and General Population

\begin{tabular}{|c|c|c|}
\hline Demographic Factors & DS Sample & General Population Estimates* \\
\hline Age, years & $\mathrm{n}=2342$ & $\mathrm{n}=244,652,666$ \\
\hline 18 to 29 & $652(27.84 \%)$ & $53,199,212(21.74 \%)^{\dagger}$ \\
\hline 30 to 39 & $406(17.34 \%)$ & $41,412,561(16.93 \%)$ \\
\hline 40 to 49 & $437(18.66 \%)$ & $41,412,561(16.93 \%)$ \\
\hline 50 to 59 & $553(23.61 \%)$ & $43,642,468(17.84 \%)$ \\
\hline 60 to 69 & $268(11.44 \%)$ & $34,085,723(13.93 \%)$ \\
\hline 70 to 79 & $26(1.11 \%)$ & $19,113,489(7.81 \%)$ \\
\hline $80+$ & $0 \%$ & $11,786,652(4.82 \%)$ \\
\hline Sex & $\mathrm{n}=2342$ & $\mathrm{n}=245,736,019$ \\
\hline Male & $1085(46.33 \%)$ & $119,945,724(48.60 \%)(>=18)$ \\
\hline Female & $1257(53.67 \%)$ & $125,790,295(51.40 \%)(>=18)$ \\
\hline Race & $\mathrm{n}=2342$ & $\mathrm{n}=318,558,162$ \\
\hline Caucasian & $1419(80.03 \%)$ & $233,657,078(73.30 \%)$ \\
\hline African American & $151(8.52 \%)$ & $40,241,818(12.60 \%)$ \\
\hline American Indian/Alaska Native & $1(0.06 \%)$ & $2,597,817(0.80 \%)$ \\
\hline Asian & $36(2.03 \%)$ & $16,614,625(5.20 \%)$ \\
\hline Other ${ }^{\ddagger}$ & $166(9.36 \%)$ & $25,446,824(8.10 \%)$ \\
\hline Ethnicity & $\mathrm{n}=2342$ & $\mathrm{n}=318,558,162$ \\
\hline Hispanic or Latino & $136(7.67 \%)$ & $55,199,107(17.30 \%)$ \\
\hline Not Hispanic or Latino & $1586(89.45 \%)$ & $263,359,055(82.70 \%)$ \\
\hline Declined & $51(2.88 \%)$ & - \\
\hline
\end{tabular}

*The 2016 entire general population $(n=318,558,162)$ was provided, with associated percentages across described age groups. These percentages were used to calculate raw numbers and adjusted percentages of adults $\geq 18$ years only $(n=244,652,666)$. Race and ethnicity represent the entire general population, including youth.

${ }^{>\dagger}$ General population heart disease, stroke and associated risk condition prevalence throughout this paper represent adults ages $\geq 20$ years. Adults ages 20 to 29 years make up $18.23 \%$, or 44,598,142 individuals, of the general adult population.

${ }^{\ddagger}$ Other represents individuals who identified as "Unknown" or "Declined" among DS sample and as "Native Hawaiian and Other Pacific Islander," "Some Other Race" or "Two or More Races" among general population. The following results will describe OR with associated $P$-values comparing prevalence of the following heart disease-related conditions among adults with DS to the general population: Coronary heart disease (CHD), Angina, myocardial infarction (MI), and HF, Stroke, diabetes mellitus (DM), Hypertension, Overweight Status, and High total cholesterol (TC). See Table 2 for complete results and Figure 1 for a mapping of the odds ratios (ORs). 
Table 2. Prevalence among Down syndrome (DS) Sample and General Population

\begin{tabular}{|c|c|c|c|c|}
\hline Clinical Disease Conditions & DS & General Population & OR (95\% CI) & $P$-Value \\
\hline CHD (includes angina and MI) & $9 / 2342(0.38 \%)$ & $18,200,000 / 271,641,791(6.70 \%)$ & $0.05(0.03,0.10)$ & $<.0001^{*}$ \\
\hline Angina & $2 / 2342(0.09 \%)$ & $9,400,000 / 261,111,111(3.60 \%)$ & $0.02(0.01,0.09)$ & $<.0001^{*}$ \\
\hline MI & $1 / 2342(0.04 \%)$ & $8,400,000 / 280,000,000(3.00 \%)$ & $0.01(0.00,0.10)$ & $<.0001^{*}$ \\
\hline Heart failure & $33 / 2342(1.41 \%)$ & $6,200,000 / 281,818,181(2.20 \%)$ & $0.64(0.45,0.90)$ & $.01 \dagger$ \\
\hline Stroke & $69 / 2342(2.95 \%)$ & $7,000,000 / 280,000,000(2.50 \%)$ & $1.18(0.93,1.50)$ & .17 \\
\hline \multicolumn{5}{|l|}{ Risk conditions } \\
\hline $\mathrm{DM}$ & $117 / 2342(5.00 \%)$ & $26,000,000 / 265,306,122(9.80 \%)$ & $0.48(0.40,0.58)$ & $<.0001^{\dagger}$ \\
\hline Hypertension & $63 / 2342(2.69 \%)$ & $116,400,000 / 253,043,478(46.0 \%)$ & $0.03(0.03,0.04)$ & $<.0001^{\dagger}$ \\
\hline Overweight (BMI, 25.0 to $29.9 \mathrm{~kg} / \mathrm{m}^{2 \ddagger}$ ) & $1332 / 1814(73.43 \%)$ & $157,232,115 / 226,559,242(69.40 \%)$ & $1.22(1.10,1.35)$ & $.00^{\dagger}$ \\
\hline Obesity (BMI, $30.0+\mathrm{kg} / \mathrm{m}^{2 \ddagger}$ ) & $780 / 1814(43.00 \%)$ & $82,241,005 / 226,559,242(36.30 \%)$ & $1.32(1.21,1.45)$ & $<.0001^{\dagger}$ \\
\hline High TC (TC $\geq 240$ mg/dL) & $63 / 2342(2.69 \%)$ & $28,500,000 / 243,589,743(11.70 \%)$ & $0.21(0.16,0.27)$ & $<.0001^{\dagger}$ \\
\hline
\end{tabular}

BMI, body mass index; CHD, coronary heart disease; DM, diabetes mellitus; MI, myocardial infarction; TC, total cholesterol; OR, odds ratio; CI, confidence interval.

*Indicates statistical significance at $P<.05$ using Fisher's Exact test.

${ }^{\dagger}$ Indicates statistical significance at $P<.05$ using Pearson's chi-square test.

${ }^{\ddagger}$ General population statistics for these conditions represent 2011 to 2014 data instead of 2013 to 2016 data.

\section{HF: Heart Failure ICD-9 428; ICD-10 150}

Adults in the DS population have 0.3647 lesser odds of experiencing HF compared with the general population $(P=.0091)$, highlighting $1.57: 1$ odds of experiencing HF among adults in the general population compared with adults with DS.

\section{Stroke: Stroke (Cerebrovascular Disease) (ICD-9 430 to 438; ICD-10 I60-I69)}

Adults in the DS population have 1.1839 greater odds of experiencing stroke compared with the general population, which is not statistically significant at $P<.05 \quad(P=.1666)$. The odds of experiencing stroke are reduced among adults in the general population compared with adults with DS (0.84:1).

\section{Hypertension: High Blood Pressure (ICD-9 401 to 404; ICD-10 I10-I15)}

Adults in the DS population have 0.9475 lesser odds of experiencing Hypertension compared with the general population $(P<.0001)$, highlighting 30.82:1 odds of experiencing hypertension among adults in the general population compared with adults with DS.

\section{Diabetes Mellitus (DM): Diabetes Mellitus (ICD-9 250; ICD-10 E10-E14)}

Adults in the DS population have 0.5160 lesser odds of experiencing DM compared with the general population $(P<.0001)$, highlighting 2.07:1 odds of experiencing DM among adults in the general population compared with adults with DS.
Overweight Status: Overweight and Obesity

Adults in the DS population have 1.2185 greater odds of being overweight or obese (Body Mass Index $(\mathrm{BMI}) \geq 25.0 \mathrm{~kg} / \mathrm{m}^{2}$ ) and 1.3238 greater odds of being obese compared with the general population $(P=.0002$ and $P<.0001$, respectively). The odds of being classified as overweight, including obese, or classified as strictly obese among adults in general population are reduced compared with adults with DS (0.82:1 and 0.76:1, respectively).

\section{High Total Cholesterol (TC): High Blood} Cholesterol and Other Lipids

Adults in the DS population have 0.7914 lesser odds of having high TC (TC $\geq 240 \mathrm{mg} / \mathrm{dL})$ compared with the general population $(P<.0001)$, highlighting 4.79:1 odds of high TC among adults in the general population compared with adults with DS.

\section{Discussion}

Research suggests that prevalence of heart disease and associated risk conditions in adults with DS is markedly different from that of those not living with DS and this study further confirms this. ${ }^{9,11}$ Among the adults with DS in our sample, prevalence of $\mathrm{CHD}$, angina, $\mathrm{MI}$, and $\mathrm{HF}$ were all statistically less when compared with the general population. In addition, hypertension, DM, and high TC were statistically less prevalent while overweight status and obesity were more prevalent. 
Figure 1. Odds ratio (OR) of condition among Down syndrome (DS) sample relative to general population. $* O R=1$ : Likelihood of condition in DS sample is the same as the likelihood of condition in general population. *OR $>1$ : Likelihood of condition in DS sample is greater than the likelihood of condition in general population. ${ }^{*} \mathrm{OR}<1$ : Likelihood of condition in DS sample is lesser than the likelihood of condition in general population. Abbreviations: CHD, Coronary heart disease; MI, myocardial infarction; DM, diabetes mellitus; BMI, body mass index; TC, total cholesterol.

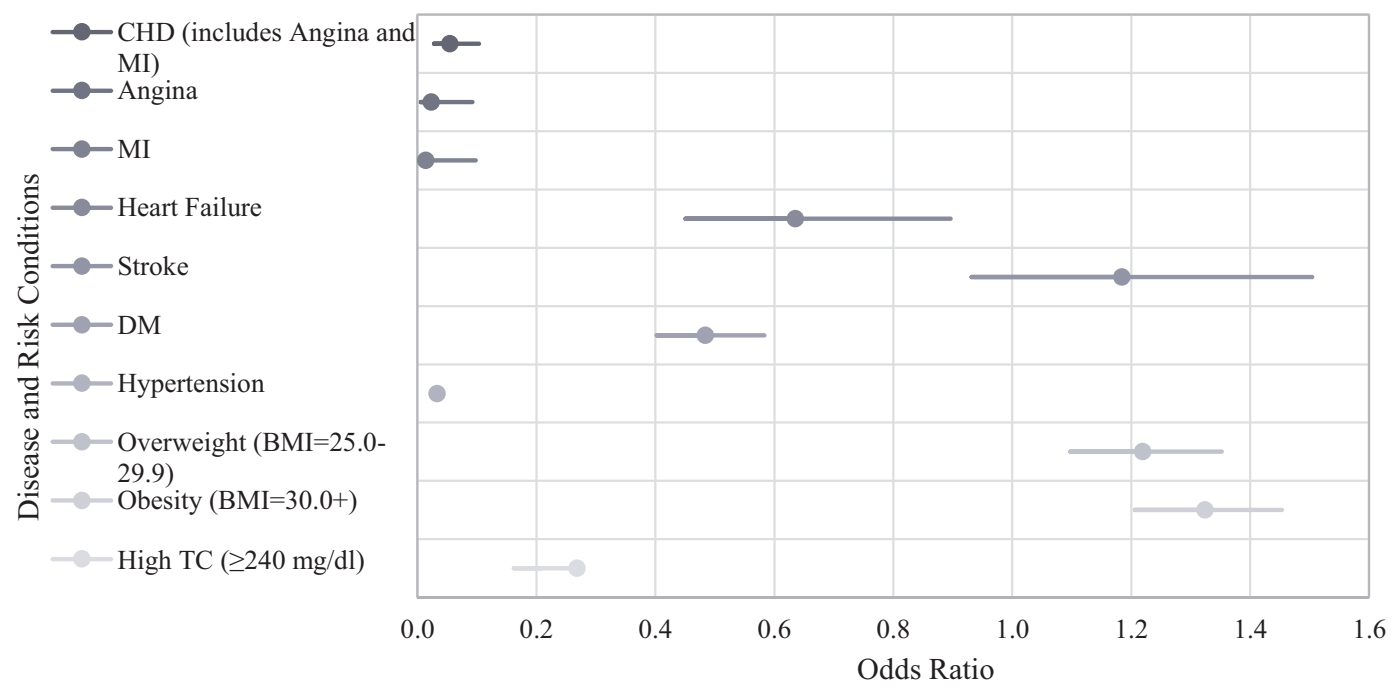

Adults with DS were as likely to experience stroke as the general population.

The AHA Report provides an annual update on heart disease-related conditions due to their health and economic burden among the US population. Obesity, in particular, is associated with a shorter lifespan and greater proportion of life lived with CVD, and a higher BMI is associated with a significantly higher risk of death attributable to CVD. ${ }^{21}$ Overweight status and obesity have also been linked with a range of chronic heart diseases, including diabetes, hypertension, CHD, and stroke; therefore, reducing obesity rates is a targeted clinical care concern among providers treating the general population. ${ }^{23}$ However, our findings suggest that the current clinical care pathway to reduce risk and prevent heart disease may not hold true, or be necessary, for adults with DS.

The potential clinical implications of this study are striking. Prevalence of heart disease and associated risk conditions in the general population look entirely different in the DS population. Therefore, clinical care guidelines should also look different. For example, common clinical targets of weight loss and prescriptions to treat high cholesterol among the general population may be ineffective when used to mitigate risk of heart disease and
CVD events among the DS population. It is hard to say what the number needed to treat would be for these conditions in this subpopulation but one could expect that it would be a much greater number with the prevalence of these conditions being so low. Although much more research is needed, the idea that treatment of an adult with DS would mirror that of adults without DS should be questioned. This study shines light on the fact that accepted treatment guidelines in the United States may not be applicable to this subgroup of adults.

Preliminary research conducted by this study's authors looking at type I and type II DM separately indicates that prevalence of type I DM and type II DM among adults with DS are 13/2342 (0.56\%) and 103/2342 (4.40\%), respectively; however, corresponding prevalence data breaking down DM type representing the general population was not available in the AHA Report. How each type of DM is related to heart disease among adults with DS should be further explored. Regarding Atherosclerosis, prior research has called for a closer look into the role of this risk factor in heart disease, as it has been found to be lower among adults with DS. ${ }^{16-18}$

Future research with a larger population of adults with DS should confirm prevalence of heart disease, stroke, and associated risk conditions. In 
addition, a larger population of adults with DS should be compared with not only the general population but matched controls, to validate and strengthen the differences between the health conditions observed among adults with DS and the general population. Furthermore, looking at heart disease among adults with DS while matching with controls without DS would also strengthen and validate this study's findings.

\section{Strength and Limitations}

Our study utilized a large patient cohort, considering the size of the sample population, thus offering some of the most robust external validity in the Unites States among studies currently available. Although the overall sample size is small relative to the number of individuals thought to have DS in the United States, the results can be generalizable to the larger DS population based on population characteristics and the geographical breadth represented by patients with DS treated by our center. Due to the limited extent of research in the field, this study will offer updated information on population health with regard to the adult DS population in the Unites States.

It should be noted that the DS sample represents prevalence of conditions across 20 years (1996 to 2016), while the general population data represents prevalence of conditions across 4 years (2012 to 2016). Considering this, the lesser prevalence of conditions among the DS sample, despite the greater number of years represented, further highlights how little individuals with DS are experiencing heart disease-related conditions.

Another important issue to note regarding this study is that the DS sample seems younger in age compared with the general population. Age among the DS sample was calculated at each case's baseline, or entry into the study. Considering the 20 years of available data, it may have been more accurate to describe each case's age at their last encounter; however, each case's age at their last encounter was unavailable.

As mentioned earlier, the current life expectancy among individuals with DS is 60 years, which is less than the average life expectancy among the general US population. Since heart disease, stroke and associated risk factors seem later in life, this is a potential limitation of this study. However, a calculated measure of association shows that the odds of being ages 40 to 79 years in this study is not statistically significantly different between the DS sample and the general population $(\mathrm{OR}=0.9340 \quad[0.8610$, 1.1032]; $P=.0999$ ), indicating that the same proportion of individuals within each group is in this older age group and thus should reflect similar prevalences of conditions if they exist.

One limitation of this study includes the methods by which data were identified before the first data abstraction. That is, DS was extracted based on a primary ICD diagnosis code of DS, possibly limiting cases to those where the primary presenting issue was directly related to DS, most commonly coded as such during physicals and other wellness check-ups; therefore, this study may have missed patients who would otherwise contributed to the representativeness of this study.

In addition, related to data abstraction, missing clinical data across health conditions is widely accepted as "not applicable" and was as such in this study, but there is a possibility that data were missing for other reasons, potentially distorting our prevalence findings. Furthermore, this study relied on ICD-9 and ICD-10 codes to assess prevalence of the conditions of focus. While medical record system ICD codes are a widely-used and accepted way of quantifying health conditions among patients, the use of ICD codes relies on clinicians to correctly and comprehensively identify conditions for coding, thus allowing for potential of underreporting of appropriate diagnoses.

The authors thank Ina Zamfirova, Suela Sulo and Laura Chicoine for early contributions to this project.

To see this article online, please go to: http://jabfm.org/content/ 33/6/923.full.

\section{References}

1. Bull MJ. Health supervision for children with Down syndrome. Pediatrics 2011;128:393-406.

2. Zhu JL, Hasle H, Correa A, Schendel D, Friedman JM, Olsen J, et al. Survival among people with Down syndrome: a nationwide population-based study in Denmark. Genet Med 2013;15:64-9.

3. Kazemi M, Salehi M, Kheirollahi M. Down syndrome: current status, challenges and future perspectives. Int J Mol Cell Med 2016;5:125-33.

4. Karam SM, Riegel M, Segal SL, Félix TM, Barros AJD, Santos IS, et al. Genetic causes of intellectual disability in a birth cohort: a population-based study. Am J Med Genet A 2015;167:1204-14. 
5. Centers for Disease Control and Prevention (CDC). Data and statistics on Down Syndrome. Updated September 19, 2019. Available from: https://www. cdc.gov/ncbddd/birthdefects/downsyndrome/data. html. Accessed October 11, 2019.

6. National Down Syndrome Society (NDSS). Down syndrome facts. National Down Syndrome Society. 2019. Available from: https://www.ndss.org/aboutdown-syndrome/down-syndrome-facts/. Accessed October 11, 2019.

7. Englund A, Jonsson B, Zander CS, Gustafsson J, Annerén G. Changes in mortality and causes of death in the Swedish Down syndrome population. Am J Med Genet A 2013;161A:642-9.

8. Glasson EJ, Sullivan SG, Hussain R, Petterson BA, Montgomery PD, Bittles AH. The changing survival profile of people with Down's syndrome: Implications for Genetic Counselling. Clin Genet 2002;62:390-3.

9. Torr J, Strydom A, Patti P, Jokinen N. Aging in Down syndrome: morbidity and mortality. J Policy Pract Intellect Disabil 2010;7:70-81.

10. Beange H, McElduff A, Baker W. Medical disorders of adults with mental retardation: a population study. Am J Ment Retard 1995;90:595-604.

11. Alexander M, Petri H, Ding Y, Wandel C, Khwaja $\mathrm{O}$, Foskett N. Morbidity and medication in a large population of individuals with Down syndrome compared to the general population. Dev Med Child Neurol 2016;58:246-54.

12. Bell AJ, Bhate MS. Prevalence of overweight and obesity in Down's syndrome and other mentally handicapped adults living in the community. J Intell Disabil Res 2008;36:359-64.

13. Prasher VP. Overweight and obesity amongst Down's syndrome adults. J Intellect Disabil Res 1995;39:437-41.

14. Melville CA, Cooper SA, McGrother CW, Thorp CF, Collacott R. Obesity in adults with Down syndrome: a case-control study. J Intellect Disabil Res 2005;49:125-33.

15. Hsieh K, Hilgenkamp T, Murthy S, Heller T, Rimmer J. Low levels of physical activity and sedentary behavior in adults with intellectual disabilities. Int. IJERPH 2017;14:1503.

16. Sobey CG, Judkins CP, Sundararajan V, Phan TG, Drummond GR, Srikanth VK. Risk of major cardiovascular events in people with Down syndrome. PLoS One 2015;10:e137093.

17. Murdoch JC, Rodger JC, Rao SS, Fletcher CD, Dunnigan MG. Down's syndrome-An atheroma free model. Br Med J 1977;2:226-8.

18. Brattstrom L, England E, Brun A. Does Down syndrome support homocysteine theory of arteriosclerosis? Lancet 1987;329:391-2.

19. Ylä-Herttuala S, Luoma J, Nikkari T, Kivimaki T. Down's syndrome and atherosclerosis. Atherosclerosis 1989;76:269-72.

20. Yang Q, Rasmussen SA, Friedman JM. Mortality associated with Down's syndrome in the USA from 1983 to 1997: a population-based study. Lancet 2002;359:1019-25.

21. Benjamin EJ, Muntner P, Alonso A, Bittencourt MS, Callaway CW, Carson AP, et al. Heart disease and stroke statistics-2019 update. Circulation 2019;139:e56-e528.

22. United States Census Bureau. American fact finder ACS demographic and housing estimates: 20122016 American Community Survey 5-year estimates. Updated 2016. Available from: https:// factfinder.census.gov/faces/tableservices/jsf/pages/ productview.xhtml? src=CF. Accessed October 9, 2019.

23. Penney TL, Kirk SFL. The health at every size paradigm and obesity: missing empirical evidence may help push the reframing obesity debate forward. Am J Public Health 2015;105:e38-e42. 
Appendix. International Classification of Diseases (ICD) Codes Defining Clinical Disease Conditions

\begin{tabular}{llc}
\hline Clinical Disease Condition & ICD-9 Code & ICD-10 Code \\
\hline Coronary heart disease (CHD) & I20-I25.9 \\
Myocardial infarction (MI) & $414.10-414.9,429.2$ & I21-I22 \\
Angina & $410.00-410.92$ & I20.9 \\
Heart failure & $413.0-413.1,413.9$ & I50 \\
Stroke (cerebrovascular disease) & 428.0 & I60-I69 \\
Risk conditions & $430-438$ & I10-I15 \\
Hypertension & $401.0-401.1,401.9,405.19,405.99$ & E10-E14 \\
Diabetes mellitus (DM) & $250.00-250.99$ & - \\
Overweight status* & - & - \\
Elevated Total Cholesterol (TC) $(\geq 240 \mathrm{mg} / \mathrm{dL})$ & & -
\end{tabular}

ICD, International Classification of Diseases.

${ }^{*} \mathrm{BMI}$ values $\geq 25.0$ was used to define overweight status, not ICD codes. 